\title{
On Certain Optimal Quadrature Formulas
}

\author{
Seymour Haber \\ Institute for Basic Standards, National Bureau of Standards, Washington, D.C. 20234
}

(October 22, 1970)

\begin{abstract}
A class of optimal quadrature formulas defined by V. L. N. Sarma in a probabilistic context is shown to be identical with a class of formulas defined previously, in a different manner, by P. J. Davis. As a result a contrast is drawn between the maximum of the error of a quadrature fomula over the unit sphere in a certain function space, and its average-in Sarma's sense-over that sphere.
\end{abstract}

Key words: Analytical functions; approximation; Gaussian quadrature; integration; optimal quadrature; quadrature.

A few years ago V. L. N. Sarma $[1]^{1}$ defined a probability measure on a certain space of functions and proposed that quadrature formulas be studied in terms of their average error on this space. If

$$
Q(f)=Q_{N}(f)=\sum_{r=1}^{N} a_{r} f\left(x_{r}\right)
$$

is any $N$-point quadrature formula, and $E^{Q}(f)=I(f)-Q(f)$ where $I(f)$ is the integral which $Q$ is approximating, we shall denote by " $\sigma\left(E^{Q}\right)$ " the "RMS" average of $E^{Q}$ with respect to Sarma's measure. Sarma calls a formula $Q_{N}$ "completely optimal" if $\sigma\left(E^{Q_{N}}\right) \leqslant \sigma\left(E^{Q_{N}^{\prime}}\right)$ for every $N$-point formula $Q_{N}^{\prime}$; and he calls it "optimal in the weights" if $\sigma\left(E^{Q_{N}}\right) \leqslant \sigma\left(E^{Q_{N}^{\prime}}\right)$ for every $N$-point $Q_{N}^{\prime}$ using the same points $x_{1}, x_{2}, \ldots ., x_{N}$. It is the purpose of this note to point out that, in the case of integration of functions of one variable, Sarma's optimal formulas are identical with those defined earlier by P. J. Davis in a quite different manner; and to discuss some consequences of this identity.

In the one-variable case, Sarma's space consists of all those functions analytic in the open unit disk whose Taylor series

$$
f(z)=\sum_{n=0}^{\infty} a_{n} z^{n}
$$

satisfy the condition

$$
\sum_{n=0}^{\infty}\left|a_{n}\right|=1
$$

The integral considered is

$$
I(f)=\int_{-1}^{1} f(x) d x
$$

AMS Subject Classification: Primary 6555, Secondary 4140.

${ }^{1}$ Figures in brackets indicate the literature references at the end of this paper. 
and he showed that the average error $\sigma$ is given by the formula

$$
\sigma^{2}\left(E^{Q}\right)=\sum_{n=0}^{\infty} 3^{-n-1}\left(E^{Q}\left(x^{n}\right)\right)^{2}
$$

([1], p. 610).

In 1953, P. J. Davis [2] introduced another measure of quadrature error for analytic functions. In the notation of [3], Davis considers the integral (4) for $f$ belonging to the Hilbert Space $H^{2}\left(C_{R}\right)$ : the space of all those functions analytic in the disk $|z|<R$ (where $R$ is any number $>1$ ) whose Taylor series satisfy the condition

$$
\sum_{n=0}^{\infty}\left|a_{n}\right|^{2} R^{2 n}<\infty
$$

The inner product is defined by

$$
(f, g)_{R}=2 \pi R \quad \sum_{n=0}^{\infty} a_{n} \bar{b}_{n} R^{2 n}=\int_{C_{R}} f(z) \overline{g(z)} d s
$$

where $a_{n}$ and $b_{n}$ are the Taylor coefficients of $f$ and $g$ respectively and $C_{R}$ is the circle $|z|=R$; $s$ denotes arc length. For any $Q$ of the form (1), with quadrature points $x_{r}$ inside $C_{R}, E^{Q}$ turns out to be a bounded linear functional, and its norm is given [3] by

$$
\left\|E^{Q}\right\|_{R}^{2}=\frac{1}{2 \pi R} \sum_{n=0}^{\infty} R^{-2 n}\left(E^{Q}\left(x^{n}\right)\right)^{2} .
$$

A knowledge of this norm affords us the error bound

$$
|I(f)-Q(f)| \leqslant\left\|E^{Q}\right\| \cdot\|f\|
$$

and Davis defined optimality of quadrature formulas in the same manner as Sarma, with the norm $\left\|E^{Q}\right\|$ in place of $\sigma\left(E^{Q}\right)$.

Comparison of (5) and (8) shows that

$$
\sigma\left(E^{Q}\right)=\left(\frac{2 \pi}{3^{1 / 2}}\right)^{1 / 2}\left\|E^{Q}\right\|_{\sqrt{3}}
$$

since the two measures of $E^{Q}$ differ by a constant factor, it follows that the quadrature formulas optimal in Sarma's sense are precisely those optimal in Davis' sense for the space $H^{2}\left(C_{\sqrt{3}}\right)$.

The $H^{2}$ spaces have many convenient analytic properties, and the norms $\left\|E^{Q}\right\|_{R}$ have been studied for various $Q$ 's ([4], [5]). In [3] I showed that for $Q=G_{N}-$ the $N$-point Gauss-Legendre quadrature formula (for a description of these formulas see, e.g., [6], chap. 8) - we have the bound

$$
\left\|E^{i_{N}}\right\|_{R} \leqslant A \sqrt{2 N+1} \rho^{-N}
$$

where

$$
\rho=2 R^{2}-1+2 R\left(R^{2}-1\right)^{1 / 2}
$$

and

$$
A=32 e^{1 / 2} \pi^{-3 / 2} \rho^{-1 / 4}\left(\frac{\rho^{1 / 2}+\rho^{-1 / 2}}{\rho^{1 / 2}-\rho^{-1 / 2}}\right)^{1 / 2} .
$$


If $R=\sqrt{3}$, then $\rho=5+2 \sqrt{6}$, and it follows that $\left\|E^{G_{N}}\right\| \sqrt{3}$ goes to zero about as fast as $(0.1010)^{N}$, or faster. In a recent paper [7], Sarma and A. H. Stroud calculated $\sigma\left(E^{G_{N}}\right)$ for $N=2,3,4, \ldots$, 20 , and observed that $\sigma\left(E^{i_{N}}\right) / \sigma\left(E^{G_{N-1}}\right)$ was very close to 0.10 . Because of the relation (9), this ratio is the same as

$$
\left\|E^{G_{N}}\right\|_{\sqrt{3}} /\left\|E^{G_{N-1}}\right\|_{\sqrt{3}}
$$

and these data seem to indicate that the left side of (10) does, indeed, give about the right rate of decrease for $\left\|E^{G_{N}}\right\|_{R}$.

(Sarma and Stroud remark that $\sigma\left(E^{G_{N}}\right) / \sigma\left(E^{G_{N-1}}\right)$ appears to be approaching 0.1 as $N \rightarrow \infty$. A closer look at their data suggests that the limit is somewhat higher. If one calculates the first differences of the successive values of $\sigma\left(E^{G_{N}}\right) / \sigma\left(E^{G_{N-1}}\right)$, it is seen that these differences are positive and decreasing, and the ratios of successive differences are increasing. If this behavior continues for higher $N, \sigma\left(E^{G_{N}}\right) / \sigma\left(E^{G_{N-1}}\right)$ must eventually become greater than

$$
\sigma\left(E^{G_{20}}\right) / \sigma\left(E^{G_{19}}\right)+\Delta_{20}\left[\left(\frac{\Delta_{20}}{\Delta_{19}}\right)+\left(\frac{\Delta_{20}}{\Delta_{19}}\right)^{2}+\left(\frac{\Delta_{20}}{\Delta_{19}}\right)^{3}+\ldots\right]
$$

where

$$
\Delta_{N}=\sigma\left(E^{G_{N}}\right) / \sigma\left(E^{G_{N-1}}\right)-\sigma\left(E^{G_{N-1}}\right) / \sigma\left(E^{G_{N-2}}\right) .
$$

The quantity (13) is greater than 0.1003 ; so it appears that the Sarma-Stroud data are consistent with the ratio of the $\sigma$ 's approaching $\left.(5+2 \sqrt{6})^{-1}\right)$.

It is interesting to compare Sarma's $\sigma$ with a very directly related error bound. Following Sarma, let $S$ be the space of all those functions analytic in the open unit disk whose Taylor series (2) satisfy

$$
\sum_{n=0}^{\infty}\left|a_{n}\right|<\infty
$$

and define

$$
\|f\|_{S}=\sum_{n=0}^{\infty}\left|a_{n}\right|
$$

$S$ is a Banach Space, isomorphic to the space $l_{1}$ of absolutely convergent series of complex numbers. Sarma's $\sigma\left(E^{Q}\right)$ is a root-mean-square average of $E^{Q}$ over the unit sphere in $S$. In contrast, the norm of $E^{Q}$ - regarded as a functional on $S$-is the supreme of $\left|E^{Q}\right|$ on the same unit sphere. This norm, which I shall denote by " $\left\|E^{Q}\right\|_{S}$ " allows the error bound

$$
|I(f)-Q(f)| \leqslant\left\|E^{Q}\right\|_{S} \cdot\|f\|_{S}, \quad f \epsilon S .
$$

In the spirit of Davis' approach, $\left\|E^{Q}\right\|_{S}$ would be used in place of $\sigma\left(E^{Q}\right)$. Let us compare the sizes of these two quantities:

For every $R>1$, if $f \epsilon H^{2}\left(C_{R}\right)$ then $f \epsilon S$; and if we write

$$
\|f\|_{R}=(f, f)_{R}^{1 / 2}=\left\{2 \pi R \sum_{n=0}^{\infty}\left|a_{n}\right|^{2} R^{2 n}\right\}^{1 / 2},
$$

we may compare $\|f\|_{R}$ and $\|f\|_{S}$. Setting $b_{n}=\left|a_{n}\right| R^{n}$, we have

$$
\begin{gathered}
\left(\sum_{n=0}^{\infty}\left|a_{n}\right|\right)^{2}=\left(\sum_{n=0}^{\infty} b_{n} R^{-n}\right)^{2} \\
\leqslant\left(\sum_{n=0}^{\infty} b_{n}^{2}\right)\left(\sum_{n=0}^{\infty} R^{-2 n}\right)
\end{gathered}
$$




$$
\leqslant \frac{R^{2}}{R^{2}-1} \sum_{n=0}^{\infty} b_{n}^{2}
$$

by the Schwartz inequality; and it follows that

where

$$
\|f\|_{S} \leqslant K(R)\|f\|_{R}
$$

$$
K(R)=\left(\frac{R}{2 \Pi\left(R^{2}-1\right)}\right)^{1 / 2}
$$

Therefore

$$
\begin{gathered}
\left\|E^{Q}\right\|_{S}=\sup _{f \in S} \frac{\left|E^{Q}(f)\right|}{\|f\|_{S}} \\
\geqslant \sup _{f \in S} \frac{\left|E^{Q}(f)\right|}{K(R)\|f\|_{R}} \\
\geqslant \frac{1}{K(R)_{f \in H_{2}\left(C_{r}\right)}} \sup ^{\left|E^{Q}(f)\right|} \\
\geqslant \frac{1}{K(R)}\|\|_{R}
\end{gathered}
$$

The experimental evidence seems to indicate that, for $Q=G_{N}$, the asymptotic behavior of $\left\|E^{G_{N}}\right\|_{R}$ for large $N$ is correctly given (at least to within factors like $N^{a}$ ) by the right side of (10). If this is so, then (20) shows that $\left\|E^{G_{N}}\right\|_{S} \neq 0\left(C^{-N}\right)$ for any $C>1$, while (by (9), (10), and (11)) $\sigma\left(E^{G_{N}}\right.$ ) $=0\left(3^{-N} N^{1 / 2}\right)$. Equation (16) may be a very conservative bound; in numerical experiments [8] an analogous bound has been found to be considerably larger than the actual quadrature error. Since Sarma's "average error" $\sigma\left(E^{G_{N}}\right)$ appears to be very much smaller than the right side of (16), it would be interesting to know whether it does, indeed, represent the "average" behavior of quadrature formulas for the integrands arising in the course of practical numerical work. I do not see how to answer this question.

\section{References}

[1] Sarma, V. L. N., Eberlein measure and mechanical quadrature formulae. I: Basic theory. Math. Comp. 22, 607-616 (1968).

[2] Davis, P. J., Errors of numerical approximation for analytic functions. J. Rat. Mech. and Anal. 2,303-313 (1953).

[3] Haber, S., The error in numerical integration of analytic functions, to appear.

[4] Hämmerlin, G., Über ableitungsfreie Schranken für Quadraturfehler, Num. Math. 5, 226-233 (1963).

[5] Barnhill, R. E., Asymptotic properties of minimum norm and optimal quadratures, Num. Math. 12, 384-393 (1968).
[6] Hildebrand, F. B., Introduction to Numerical Analysis (McGrawHill N.T., 1956).

[7] Sarma, V. L. N., and Stroud, A. H., Eberlein measure and mechanical quadrature formulae. II: Numerical results, Math. Comp. 23, 781-784 (1969).

[8] Barnhill, R. E., Optimal quadratures in $L^{2}\left(E_{p}\right)$. I. SIAM J. Numer. Anal. 4, 390-397 (1967).

(Paper 75B1\&2-348) 